\title{
Latest Advances in Concepts and Treatment Protocols of Dental Implants: A Brief Review
}

\author{
${ }^{1}$ Hasan Sarfaraz, ${ }^{2}$ Saurabh Chhabra \\ ${ }^{1}$ Professor, Department of Prosthodontics, Yenepoya Dental College, Yenepoya University, Deralakatte, Mangalore, Karnataka, India \\ ${ }^{2}$ Senior Resident, NDMC Hospital, New Delhi, India
}

Correspondence: Hasan Sarfaraz, Professor, Department of Prosthodontics, Yenepoya Dental College, Yenepoya University Deralakatte, Mangalore-575018, Karnataka, India, e-mail: hsarfaraz@hotmail.com

\begin{abstract}
The long-term success of an implant depends on the stability of bone support for the implant. Implantology has evolved from the conventional protocols suggested by Branemark to more challenging and predictable approach. The purpose of this article is to present a brief review of literature on the factors that influence the success and survival rates of dental implants and also to review the newer concepts in implantology. There is enough evidence in literature that diagnosis and treatment planning must have a proven scientific basis, if consistency of results is to be achieved. Treatment planning must begin through a visualization of the end result. By paying attention to details, systematically analyzing and recognizing inadequacies in each factor predictable implant success can be achieved,

Keywords: Implant design, Implant surface topography, Implant stability, Crestal bone loss and preservation, Immediate implant placement, Progressive loading, Implant protective occlusion.
\end{abstract}

\section{INTRODUCTION}

Branemark et $\mathrm{al}^{1}$ defined osseointegration as a structural and functional connection between ordered living bone and the surface of a load carrying implant. It is this interface that is formed during the healing period and maintained throughout the postprosthetic loading period that determines the success of an implant. Development of the implant bone interface is complex and involves numerous factors. These include not only implant related factors, such as material, shape, topography and surface chemistry, but also mechanical loading, surgical technique and patient factors, such as bone quality and quantity.

A comprehensive review of the literature by Charles $\mathrm{J}$ Goodacre $^{2}$ identified the following clinical complications of osseointegrated implants: Greater implant loss occurred with over dentures than with other types of prostheses. There was greater loss in the maxilla than mandible. Implant loss increased with short implants and poor bone quality. Periimplant soft tissue complications included dehiscence and gingival inflammation/proliferation. Mechanical or prosthetic complications were screw loosening/fractures, implant fractures, framework, resin base and veneering material fractures. Some studies also presented phonetic and esthetic complication.

The purpose of this article is to present a brief review of literature on the factors that influence the success and survival rates of dental implants. Google Scholar was used to search the English language literature. The keywords used included implant success, implant stability, implant bone interface, crestal bone loss, implant design, implant surface and implant abutment interface. Most recent articles were reviewed, and additionally references were obtained from a hand search of reviewed articles.

\section{IMPLANT DESIGN}

Implant design refers to the macro and microstructure of an implant system, such as shape, type of implant abutment connection presence of thread, thread design and surface treatment.

Increasing the functional surface area of an implant will improve the way stress is distributed resulting in lesser forces at the crest. Use of threaded implants than the cylindrical implants for crestal bone preservation has been well documented in the literature.

\section{Thread Geometry}

Thread depth, thread face angle and thread pitch are some of the varying geometric patterns that determine the functional thread surface and affect the biomechanical load distribution of the implant. The influence of threads can be easily understood as the greater the numbers of threads that are present as well as greater the depth of the threads, the more functional surface area that is available. It has been found that the shear force on a V-shaped thread face that is $30^{\circ}$ is approximately 10 times greater than the shear force on a square thread. ${ }^{3}$ Therefore, square shaped threaded implants will concentrate lesser forces at crestal bone as well.

Since the 1970s until the early 1990s dental implants have smooth necks to prevent plaque accumulation, and this concept has been adopted by most dental implant manufacturers. However, this machined neck is not an 
effective design for the distribution of occlusal force. Many longitudinal studies have shown the marginal bone level to be resorbed to the first thread of machined implants after a year of function. ${ }^{4,5}$ Bone growth over the cover screw is often seen at second stage surgery, but after functional loading, bone loss down to the first thread has been noted. ${ }^{6}$

To minimize marginal bone loss, in addition to the use of a rough surface at the marginal bone level, a macroscopic modification, such as the addition of microthreads has been recommended. A rough surface and microthreads at the implant neck is believed to not only reduce crestal bone loss but also help with early biomechanical adaptation against loading in comparison to the machined neck design.

Many clinical studies have been conducted on the effect of microthread on crestal bone loss. It can be concluded from these studies that the use of a rough surface with microthreads on implant at the crest region was the most effective design to maintain the marginal bone level after functional loading.

\section{Implant Dimensions}

Increasing implant length and width increase the surface area but it has been found that implant width is more important for crestal bone preservation than the implant length as stress values and concentration areas decreased for cortical bone when implant diameter is increased. ${ }^{7}$

Shinichiro Tada et $\mathrm{al}^{8}$ did a finite element analysis in which they found that highest cortical bone stresses in all types of bone are located around the implant neck but strain distributions around the cancellous bone, showed some differences. In type 1 and type 2 cancellous bone, highest stresses were found around the implant neck but in type 3 and type 4 cancellous bones, the threads of the screw type implants effectively reduced the degree of stress, generating moderate strain in bone around thread crests and evenly distributed low strain in other regions. They concluded that increasing implant width is more beneficial for type 1 and type 2 bones and increasing implant length is more beneficial for type 3 and type 4 bones.

Winkler et $\mathrm{a}^{9}$ studied the influence of implant diameter and length on implant success rate. Their results on 3-year survival and stability of various implant lengths and diameters were $90.7 \%$ for 3 to $3.9 \mathrm{~mm}$ and $94.6 \%$ for 4 to $4.9 \mathrm{~mm}$ implants. Also, longer implants had significantly better survival rates as compared with shorter implants.

\section{Implant Surface Topography}

Efforts to enhance implant surface have focused on improving the predictability, rate and degree of osseointegration. Commonly, modification to the implant surface have been made through the use of additive methods, such as titanium plasma spraying, hydroxyapatite coating or subtractive techniques, such as grit or sandblasting with aluminous oxides and etching.
Some of the important advantages of surface roughness include increased surface area of the implant adjacent to bone, improved cell attachment to the implant surface and thus increased biomechanical interaction of the implant with bone. ${ }^{10}$ In an extensive review article, ${ }^{11}$ publications that evaluated implant use in patients were assessed to determine whether differences existed in success rates of implants with relatively smooth surfaces compared to implants having roughened surface. He concluded that rough surfaced implants had significantly higher success rates compared to implant with machined surfaces. Human histological studies have demonstrated improved bone implant connection on rough surfaced implants compared to smooth surfaced implants. ${ }^{12}$ There is clear evidence that rough surfaced implants decrease the integration time and may decrease overall treatment time appreciably.

\section{IMPLANT ABUTMENT INTERFACE}

The implant abutment connection can be an area where adverse biologic and mechanical failures can occur. Biological complications, such as increased microleakage, gingivitis and bone loss, have been reported to result from poorly adapted implant abutment interface. Mechanical complications, such as increased incidences of abutment rotation and breakage, screw loosening, have also been reported.

In implant dentistry, there are two basic approaches to place endosseous implants that is submerged where in the coronal portion of the implant is at or below the level of alveolar crest and in the non-submerged approach the top of the implants lie above the crest of bone. In some of the submerged systems a "microgap" may exist at the level of the alveolar crest where the abutment and the implant meet. This microgap is usually associated with inflammation and alveolar crest bone loss. One of the prevailing hypotheses regarding this phenomenon is that oral bacteria colonize in this area following the placement of abutment, which leads to infection and thus peri-implantitis. Many studies have shown that a crestal bone loss of about $2 \mathrm{~mm}$ will occur with submerged, two-piece approach, dependent on the location of the microgap. Using a nonsubmerged, one piece implant design, showed minimal to no resorption. ${ }^{13-15}$

Stefania $\mathrm{C}$ et $\mathrm{al}^{16} \mathrm{did}$ an investigation to compare the vertical and horizontal misfit at the implant abutment interface and concluded that horizontal misfit was greater than vertical misfit in all groups, including the machined group.

Joachm S Hermann et $\mathrm{al}^{17}$ did a nondecalcified histological study on randomly placed canine mandibular titanium implants and found that the crestal bone changes around two-piece, nonsubmerged titanium implants are significantly influenced by possible movements between implants and abutments, but not by the size of the microgap (interface). Thus, significant crestal bone loss occurs in 
two-piece implant configurations even with the smallest sized microgaps $(<10 \mu \mathrm{m})$ in combination with possible movements between implant components.

Studies have shown that the internal hex implant system generated the lowest maximum Von Mises stresses for all loading conditions because of reduction of the bending effect by sliding in the tapered joints between the implant and the abutment. ${ }^{18}$ Implant system with long internal tube-in-tube connections and cam-slot fixation showed advantages with regards to longevity and fracture strength compared with systems with shorter internal or external connection designs. $^{19}$

Many studies have reported that prosthetic complications, such as screw loosening and screw fractures, have been observed in the first year postloading than in subsequent years. Studies have also proven that screw retained restorations in the posterior region are expected to have higher complications rate than cemented ones, especially during the first year of loading. Therefore, the use of screw retained prosthesis to ensure retrievability and thus facilitate reintervention may be of limited relevance. ${ }^{20}$

\section{IMPLANT STABILITY}

One of the most critical factors in successful osseointegration of an implant is stability in the bone at the time of placement, (primary stability). Any motion between the implant body and the surrounding bone during the early healing phase is considered to be a high risk factor for early implant failure. Several factors, such as implant geometry, preparation technique and quality and quantity of local bone, influence primary stability.

The high failure rates observed in type 4 bone may be explained by the fact that pretapping initially was used in all bone qualities. Because of the "soft bone problem", most surgeons today use self tapping implants or place standard implants as self-tapping implants in soft quality bone. Wider implants, smaller drill diameters are often used to confirm the bone for good primary stability. There are newer designs of implants to be used in soft bone to increase primary stability. One obvious way to increase stability after implant surgery is to allow the surrounding bone to heal before loading. Friberg et $\mathrm{al}^{21}$ concluded from the 20-month clinical study that the stability of implant in soft bone increased more than that of implant in denser bone from placement to abutment connection. Twenty months after placement, all implants had reached a similar degree fo stability irrespective of bone quality and primary stability.

Ilser Turkyilmaz and Edwin A McGlumphy ${ }^{22}$ studied the influence of bone density on implant stability parameters and implant success and concluded that significant correlations found between bone quality and implant stability parameters indicate that clinicians may predict primary stability before implant insertion, and they may modify their treatment plans (i.e. implant locations, longer healing periods) before implant surgery, where the bone quality is poor.

\section{IMPLANT LOADING PROTOCOLS}

Conventional loading protocols as envisaged by different implant systems included healing periods of typically 12 to 24 weeks. This is based on the initial clinical experience of Branemark. Clinical and experimental research directly challenged this notion with convincing outcomes. Experimental research indicated that early loading itself was not a contraindication to successful osseointegration. The latter was dependent on maintenance of a load that prevented extensive micromotion at the bone-implant interface.

Cochran et $\mathrm{al}^{23}$ recommended working definition of these loading protocols, immediate loading protocols in which the implants were loaded within 2 days of surgery and early loading protocols wherein a provisional prosthesis was inserted at a subsequent visit prior to osseointegration. Though the implants were not loaded the same day, these protocols directly challenged the healing process by introducing loading during wound healing. The time period suggested for insertion of the prosthesis was between 2 days and 3 months after surgery. In delayed loading protocols, the healing period was extended due to the compromised host site conditions and, typically, prosthesis connection is later than the conventional healing period.

Nikolai J Attard and George A Zarb, ${ }^{24}$ did an extensive literature review of clinical studies on immediate and early implant loading protocols and concluded within the limitations of the studies, only treatment protocols in the anterior mandible can result in predictable results. Limited studies on the edentulous maxilla and partially edentulous patients precluded definite conclusions. More accurate long term studies with stronger research design and reporting on treatment protocols for separate clinical situations are required to allow meaningful comparisons and conclusions. Furthermore, there is a need for research to evaluate the implications of these protocols on patient mediated outcomes.

\section{Progressive Loading}

The progressive loading of implants was first suggested by Misch in $1980 .^{25}$ A study by Manz ${ }^{26}$ found that the crestal bone loss after successful bone integration was related directly to the bone density. An implant may fail, if the stresses applied exceed the physiologic limits of the bone density present around the implant. A gradual and progressive increase in the loads during prosthetic fabrication stimulates an increase in density. This will result in definite preservation of crestal bone particularly in type 3 and type 4 bone. Misch et $\mathrm{al}^{27}$ found that periotest values are significantly reduced in progressively loaded implants placed in D 4 types of bone. Similarly Rotter BE et $\mathrm{al}^{28}$ by 
periotest studies found that there was larger increase in implant rigidity for progressively loaded implants.

Appleton et $\mathrm{al}^{29}$ evaluated peri-implant bone changes in response to progressive loading of the implants. Crestal bone loss and bone density were measured by digital substraction radiology. The mean crestal bone loss for progressively loaded implants was found to be $0.13 \mathrm{~mm}$ at 2 months, $0.18 \mathrm{~mm}$ at 4 months , $0.24 \mathrm{~mm}$ at 6 months and $0.32 \mathrm{~mm}$ at 12 months. The mean crestal bone loss for the control group was $0.31 \mathrm{~mm}$ at 2 months, $0.35 \mathrm{~mm}$ at 4 months, $0.41 \mathrm{~mm}$ at 6 months and $0.47 \mathrm{~mm}$ at 12 months. This suggested that loss of crestal bone is significantly reduced by progressive loading.

\section{Implants placed in Fresh Extraction Sites}

The rationale proposed for implant placement in fresh extraction sites was to preserve soft tissue esthetics and to further reduce the treatment time and associated costs by avoiding an intermediate stage of removable denture wear. Many studies have suggested that success was not compromised by placement in extraction sockets as long as primary stability was achieved. Nevertheless, success was reduced when implants were placed in morphologically compromised jawbone sites. ${ }^{30-33}$

De Bruyn and Collaert ${ }^{34}$ reported that $39 \%$ of machined implants placed in extraction sites failed to osseointegrate and observed that implants placed in extraction sites with a history of previous periodontal disease were more susceptible to failures. To conclude, these short- to mediumterm studies suggested that implant placement should be restricted to extraction sites without a history of periodontal disease and limited to the anterior mandible. Further longterm clinical research is required to support these observations and to determine the efficacy of a similar protocol in other jawbone sites.

\section{IMPLANT PROTECTIVE OCCLUSION}

Occlusal overload is considered a major etiological factor in implant failure. Osseointegrated implants are ankylosed to surrounding bone without the periodontal ligament which has mechanoreceptors and shock absorbing function. In addition, the crestal bone around implant could be a fulcrum point for lever action when a bending moment is applied.

Improper occlusal contacts will transfer stresses around the implant due to overload resulting in crestal bone resorption. A favorable occlusal scheme is one of the major factors in implant survival, particularly when parafunction is present. An implant protective occlusion should have:

- No premature contacts or interferences

- Mutually protective occlusion

- Least cuspal inclinations

- No cantilever or offset distance

- Occlusal contact positions along the long axis of the implant

- Proper implant crown contour
- Protection of the weakest component and the occlusal materials should be followed to minimize the overload and hence, the loss of crestal bone which ultimately prevents implant failure. ${ }^{35}$

\section{PERI-IMPLANT SOFT TISSUES}

The attachment between the mucosa and the titanium implant surface consists of a junctional epithelium (approx $2 \mathrm{~mm}$ ) and a connective tissue zone (approx $1 \mathrm{~mm}$ ). This soft tissue seal protects the zone of osseointegration from the oral cavity as well as from harmful substances produced by bacterial plaque. This characteristic, in turn, stresses on the importance of both the creation of a soft tissue anatomy around implants and proper super structure design to facilitate a high standard of oral hygiene. Such measures will minimize the frequency of an inflammatory condition of the soft and hard tissue anchoring the implant.

Berglundh and Lindhe ${ }^{36}$ studied the dimension of the mucosa-implant attachment by placing implants in dogs on the control site with mucosa of $4 \mathrm{~mm}$ and at the test site with mucosal thickness of $2 \mathrm{~mm}$. Mechanical plaque control was performed during next 6 months. Histological examinations revealed that the resulting peri-implant soft tissue seal was almost identical. At sites where the volume of the mucosa was reduced, the healing process consistently included bone resorption (angular bone loss) to create a soft tissue seal that was about $3 \mathrm{~mm}$ high. Breglundh and Lindhe ${ }^{36}$ concluded that a certain minimum width of the peri-implant mucosa is required, and that bone resorption may take place to allow a proper soft tissue attachment to form.

\section{CONCLUSION}

The replacement of missing teeth with dental implants remains a difficult task under most conditions. With comprehensive treatment planning and proper surgical and restorative protocols, satisfactory results can be achieved. Diagnosis and treatment planning must have a proven scientific basis, if consistency of results is to be achieved. Without science as our guiding light, any implant success is limited to initial gratification and ignores the far greater elements of a problematic outcome yet to occur. Treatment planning must begin through a visualization of the end result. By paying attention to details, systematically analyzing and recognizing inadequacies in each factor predictable implant success can be achieved.

\section{REFERENCES}

1. Branemark PI, Svensson B, Van Steenberghe D, Darius P. Tenyear survival rates of fixed prostheses on four or six implants ad modum Branemark in full edentulism. Clin Oral Implants Res 1995;6:227-31

2. Goodacre Charles J, Khan Joseph YK, Rungcharassaeng Kitchai. Clinical complications of osseointegrated implants. J Prosthet Dent 1999;81:537-52. 
3. Misch CE, Bidez MW. A scientific rationale for dental implant design. In: Misch CE (Ed). Contemporary implant dentistry (2nd ed). St Louis: Mosby 1999.

4. Jung YC, Han CH, Lee KW. A 1 year radiographic evaluation of marginal bone around dental implants. Int J Oral Maxillofac Implants 1996;11:811-18.

5. Oh Tae J, Yoon JK, Misch CE, Wang HL. The causes of early implant bone loss: Myth or science? J Periodontol 2002;73: 322-33.

6. Adell R, Lekhom U, Rockler B, Branemark PI. A 15-year study of osseointegrated implants in the treatment of the edentulous jaw. Int J Oral Surg 1981;10:387-416.

7. Luigi Baggi, Ilaria Cappelloni, Michele Di Girolamo, Franco Maceri, Giuseppe Vairo. The influence of implant diameter and length on stress distribution of osseointegrated implants related to crestal bone geometry: A three-dimensional finite element analysis. J Prosthet Dent 2008;100:422-31.

8. Shinichiro Tada, Roxana Stegaroiu, Eriko Kitamura, Osamu Miyakawa, Haruka Kusakari. Influence of implant design and bone quality on stress/strain distribution in bone around implants: A three-dimensional finite element analysis. Int J Oral Maxillofac Implants 2003;18:357-68.

9. Winkler S, Morris HF, Ochi S. Implant survival to 36 months as related to length and diameter. Ann Periodontol 2000;5: 22-31.

10. Cooper LF. A role for surface topography in creating and maintaining bone at endosseous implants. J Prosthet Dent 2000;84:522-34.

11. Cochran DL. A comparison of endosseous dental implants surfaces. J Periodontol 1999;70:1523-39.

12. Ivanoff CJ, Widmark G, Johansson C, Wennerberg A. Histologic evaluation of bone response to oxidized and turned titanium micro-implants in human jawbone. Int J Oral Maxillofacial Implants 2003;18:341-48.

13. Hermann JS, Cochran DL, Nummikosi PV, Busser D. Crestal bone changes around titanium implants. A radiographic evaluation of unloaded nonsubmerged and submerged implants in the canine mandible. J Periodontol 1997;68:1117-30.

14. Hermann JS, Busser D, Schenck RK, Cochran DL. Crestal bone changes around titanium implants. A histometric evaluation of unloaded nonsubmerged and submerged implants in the canine mandible. J Periodontol 2000;71:1412-24.

15. Hermann JS, Schoolfield JD, Nummikosi PV, Busser D, Schenck RK, Cochran DL. Crestal bone changes around titanium implants. A methodological study comparing linear radiographic versus histometric measurements. Intl J Oral Maxillofac Implants 2001;16:475-85.

16. Stefania C Kano, Paul P Binon, Donald A Curtis. A classification system to measure the implant-abutment microgap. Intl J of Oral Maxillofac Implants 2007;22:879-85.

17. Hermann JS, Schoolfield JD, Schenck RK, Busser D, Cochran $\mathrm{DL}$. Influence of the size of the microgap on crestal bone changes around titanium implants: A histometric evaluation of unloaded nonsubmerged implants in the canine mandible. J Periodontol 2001;72:1372-83.

18. Chun HJ, Shin HS, Han $\mathrm{CH}$, Lee SH. Influence of implant abutment types on stress distribution in bone under various loading conditions using finite element analysis. Intl J Oral Maxillofac Implants 2006;21:195-202.
19. Steinebrunner L, Wolfart S, Ludwig K, Kem M. Implant abutment interface design affects fatigue and fracture strength of implants. Clin Oral Impl Res 2008;19:1276-84.

20. Rebah Nadir, Mark Bischof, Moncler SS, Belser UC, Jacky Samson. Prosthetic complications with dental implants: From an up to 8-year experience in private practice. Int J Oral Maxillofac Implants 2006;21:919-28.

21. Friberg B, Sennerby L, Meredith N, Lekholm U. A comparison between cutting torque and resonance frequency measurements of maxillary implants: A 20-month clinical study. Int J of Max Surgery 1996;28:297-303.

22. Ilser Turkyilmaz, Edwin A McGlumphy. Influence of bone density on implant stability parameters and implant success: A retrospective clinical study. BMC Oral Health 2008;8:32.

23. Cochran DL, Morton DJ, Weber H. Consensus statements and recommended clinical procedures regarding loading protocols for endosseous dental implants. Int J Oral Maxillofac Implants 2004;19:109-13.

24. Nikolai J Attard, Zarb George A. Immediate and early implant loading protocols: A literature review of clinical studies. J Prosthet Dent 2005;94:242-58.

25. Misch CE. Gradual load on an implant restoration. Tatum implant seminars lecture. St Petersberg: Fla 1980.

26. Manz MC. DICRG interim report no 9,1997. Radiographic assessment of peri-implant bone loss. J Oral Maxillofac Surg 1997;55:76-82.

27. Misch CE. Progressive bone loading. Pract Periodontics Aesthet Dent 1990;2:27-30.

28. Rotter BE, Blackwell R, Dalton G. Testing progressive loading of endosteal implants with the periotest: A pilot study. Implant Dent 1996;5(1):28-32.

29. Appleton RS, Nummikoski PV, Pigmo MA, et al. Peri-implant bone changes in response to progressive osseous loading. J Dent Res 1996.

30. Balshi TJ, Wolfinger GJ. Immediate loading of Branemark implants in edentulous mandibles: A preliminary report. Implant Dent 1997;6:83-88.

31. Wolfinger GJ, Balshi TJ, Rangert B. Immediate functional loading of Branemark system implants in edentulous mandibles: Clinical report of the results of development and simplified protocols. Int J Oral Maxillofac Implants 2003;18:250-57.

32. Aires I, Berger J. Immediate placement in extraction sites followed by immediate loading: A pilot study and case presentation. Implant Dent 2002;11:87-94.

33. Kronstrom M, Widbom T, Lofquist LE, Henningson C, Widbom C, Lundberg T. Early functional loading of conical Branemark implants in the edentulous mandible: A 12-month follow-up clinical report. J Prosthet Dent 2003;89:335-40.

34. De Bruyn H, Collaert B. Early loading of machined-surface Branemark implants in completely edentulous mandibles: Healed bone versus fresh extraction sites. Clin Implant Dent Relat Res 2002;4:136-42.

35. Misch CE , Bidez. Occlusal considerations for implant supported prostheses: Implant protective occlusion. In: Misch CE (Ed). Dental implant prosthetics. St Louis: Mosby 2005.

36. Berglundh T, Lindhe J. Dimension of the peri-implant mucosa. Biologic width revisited. J Clin Periodontol 1996;23:971-73. 